\title{
Association of dietary patterns with sociodemographic and health-related factors among Coronary Artery Disease (CAD) patients
}

\begin{abstract}
This study aimed to identify the association of dietary patterns with sociodemographic and health-related characteristics among coronary artery disease patients. In this cross-sectional study, the participants were 250 patients coronary artery disease aged $\geq 40$ years old. Data collection was done using questionnaires related to sociodemographics, health-related factors, and food-frequency intake information. Three dietary patterns (traditional, western, and healthy) were obtained using principal component analysis. The result showed that dietary patterns were associated with sociodemographic and health-related factors. According to the result, all the factors were taken very seriously when planning a promotional program for healthy lifestyle in prevention of CAD.
\end{abstract}

Keyword: Coronary artery disease; Dietary patterns; Health-related factors; Sociodemographics 